and patients with HCC and BCA the lowest proportion (40.0\%).

Conclusions The observed findings underline the importance of TLS as a novel biomarker and a possible association to cellular responses may even enhance their prognostic value. Our planned analyses of combined humoral immune responses will further elucidate the role of TLS in antitumor immune response of the analyzed cancer types. A combined targeting of a predefined or personalized set of included TAAs appears promising across the different cancer types.

Disclosure Information E. Preugszat: B. Research Grant (principal investigator, collaborator or consultant and pending grants as well as grants already received); Modest; Koeln Fortune Program, Faculty of Medicine, University of Cologne. M. Thelen: None. M. Garcia-Marquez: None. J. Lehmann: None. D. Keller: None. M. von Bergwelt-Baildon: Other; Modest; Honoraria for advisory boards, for invited talks from BMS and financial support for research projects from Astellas, Roche and MSD. H.A. Schlößer: Other; Significant; Financial support for research projects from Astra Zeneca.

\section{P06.03 BISPECIFIC ANTIBODY-DRIVEN SYNTHETIC AGONISTIC RECEPTOR ENGINEERED T CELLS LEAD TO SPECIFIC AND CONDITIONAL THERAPY IN MELANOMA CANCER MODELS}

${ }^{1} \mathrm{M}$ Benmebarek*, ${ }^{1} \mathrm{~F}$ Märkl, ${ }^{1} \mathrm{~J}$ Keyl, ${ }^{1} \mathrm{~B}$ Loureiro Cadilha, ${ }^{2} \mathrm{M}$ Geiger, ${ }^{1} \mathrm{C}$ Karches, 'S Endres, ${ }^{1} \mathrm{C}$ Klein, ${ }^{1} \mathrm{~S}$ Kobold, ${ }^{1} \mathrm{~A}$ Klüver, ${ }^{1} \mathrm{M}$ Schwerdtfeger. ${ }^{1}$ Center of Integrated Protein Science Munich (CIPS-M) and Division of Clinical Pharmacology, Department of Medicine IV, Klinikum der Universität München, Munich, Germany; ${ }^{2}$ Roche Innovation Center Zurich, Schlieren, Switzerland

\subsection{6/jitc-2021-ITOC8.37}

Background Immunotherapeutic approaches, including immune checkpoint blockade and adoptive T cell therapy (ACT) in the form of tumor-infiltrating lymphocytes (TIL), have had marked success in the treatment of melanoma. Despite these successes, many patients are refractory to treatment or relapse with therapy-resistant disease. To overcome said limitations, we propose a controlled ACT approach, where T cells are armed with synthetic agonistic receptors (SAR) that are conditionally activated only in the presence of a target melanoma-associated antigen, and a cross-linking bispecific antibody (BiAb) specific for both SAR T cell and tumour cell.

Materials and Methods A SAR composed of an extracellular EGFRvIII, trans- membrane CD28, and intracellular CD28 and $\mathrm{CD} 3 \mathrm{z}$ domains was fused via overlap- extension PCR cloning. $\mathrm{T}$ cells were retrovirally transduced to stably express our SAR construct. We validated our approach in two murine as well as two human cancer models expressing our melanoma-associated target antigens TYRP (murine) and MCSP (human). We confirmed conditional and specific stimulation and proliferation of our $\mathrm{T}$ cells, as well as their tumour-antigen-directed cytotoxicity, in vitro and in vivo.

Results Crosslinking TYRP-EGFRvIII (murine) and MCSPEGFRvIII (human) BiAb, monovalently selective for our SAR, induced conditional antigen-dependent activation, proliferation of SAR-T cells and directed tumour cell lysis with specificity towards two TYRP-expressing murine melanoma and two MCSP-expressing human melanoma cancer models. In vivo, anti-tumoural activity was mediated by the co-administration of SAR-T cells and BiAb, in A375 and MV3 melanoma xenograft models. Further, we could show that SAR T cells exhibited resistance to MDSC-induced suppression of activation and proliferation.

Conclusions Here we apply the SAR $x$ BiAb approach in efforts to deliver specific and conditional activation of SAR transduced $\mathrm{T}$ cells, and targeted tumour cell lysis. The modularity of our platform is key for a targeting approach in a tumor entity with a high mutational load such as melanoma and is fundamental in our drive towards personalised immunotherapies. Further, the SAR approach has demonstrated resistance to MDSC-induced suppression, an interesting axis that requires further investigation.

Disclosure Information M. Benmebarek: None. F. Märkl: None. J. Keyl: None. B. Loureiro Cadilha: None. M. Geiger: None. C. Karches: None. S. Endres: None. C. Klein: None. S. Kobold: None. A. Klüver: None. M. Schwerdtfeger: None.

\section{P06.04 TRANSCRIPTOME-WIDE NETWORK ANALYSIS PREDICTS THE ROLE OF LACTATE DEHYDROGENASE C IN BREAST CANCER CELL SURVIVAL AND IMMUNE DYSFUNCTION}

A Naik* , J Decock. Qatar Biomedical Research Institute, Doha, Qatar

\subsection{6/jitc-2021-ITOC8.38}

Background Cancer testis antigens (CTAs) are lucrative anticancer targets given their restricted expression patterns and known roles as mediators of cancer hallmarks, including cancer metabolism, proliferation, survival, and cell motility. Lactate dehydrogenase $\mathrm{C}$ (LDHC) is a CTA with upregulated expression in poor prognosis subtypes of breast cancer, however its tumorigenic role is less understood. We recently reported that silencing LDHC reduces breast cancer cell survival through a dysregulated DNA damage response, thus highlighting its potential as an anti-cancer target with limited off-target effects. This study aimed to explore the changes in the transcriptome of breast cancer cells and immune-related mediators upon in vitro LDHC targeting.

Materials and Methods We silenced LDHC expression in breast cancer cell lines and investigated the downstream effects on the tumor cell transcriptome. Differentially expressed genes were subjected to regulatory network analyses. We further assessed the secretory profile of cytokines and immune checkpoint expression in LDHCsilenced cells and used the Tumor Immune Dysfunction and Exclusion (TIDE) algorithm to determine the effect of the interaction between $L D H C$ expression and cytotoxic $\mathrm{T}$ lymphocyte (CTL) infiltration in the METABRIC breast cancer cohort.

Results Network analysis to investigate the effects of silencing $L D H C$ on the tumor cell transcriptome identified 47 up- and 55 down-regulated transcripts $(2.0$-fold change, adj $\mathrm{p}<0.05)$. Differentially expressed genes in the $L D H C$-silenced cells were particularly enriched in canonical pathways regulating cell cycle checkpoint control, BRCA1-mediated DNA damage response and NF-kb signaling in response to infection. Upstream regulator analyses revealed the altered expression profile was associated with $\operatorname{mTOR}(\mathrm{p}=1.27 \mathrm{e}-5, \mathrm{z}=2.242)$ and CASP3 $(\mathrm{p}=3.2 \mathrm{e}-4, \mathrm{z}=2.250)$ pathways, which in the presence of LDHC are predicted to activate TP53, Myc, NF-KB complex, STAT1/3, PRKC, CDK2, FOXO3 and HIF-1a while 
inhibiting SMAD3, PTEN, ATM, IL18 and BCL2. Consequentially, the observed network-wide changes on LDHC silencing are predicted to negatively influence cellular growth and proliferation, cell migration and cell infiltration. The LDHC-associated network indicated a higher-level regulation by $\operatorname{miR} 378 \mathrm{a}-3 \mathrm{p} \quad(\mathrm{p}=1.4 \mathrm{e}-7, \mathrm{z}=-3.117)$, affecting the downstream mechanistic in LDHC-expressing cells. Interestingly, the miR378a causal network also indicated inhibition of the immune response in LDHC-positive cells. TIDE analysis indicated that high expression of LDHC in the METABRIC Her2 breast cancer cohort (TIDE score $=1.97, p=0.049$ ), and to a lesser extent in triple negative breast cancer (TIDE score $=0.466, \mathrm{p}=0.642$ ), decreases the beneficial effect between CTLs and overall survival observed in LDHC Low tumors. Concurrently, LDHC-silenced cells displayed a proinflammatory gene expression and cytokine profile and down-regulated the expression of PD-L1 and Gal-9 immune checkpoints.

Conclusions Our findings provide an indication of potential CTL dysfunction in breast tumors with high LDHC expression and suggests that therapeutic targeting of LDHC may inhibit tumor growth while releasing the anti-tumor immune response in breast cancer.

Disclosure Information A. Naik: None. J. Decock: None.

\section{P06.05 ENDOGENOUS T-CELL RESPONSES TO TEN MAJOR CANCER TESTIS ANTIGENS ARE FREQUENT IN ESOPHAGO-GASTRIC ADENOCARCINOMA AND ANTIGEN-SPECIFIC T CELLS CAN BE EXPANDED USING CD40-ACTIVATED B CELLS}

${ }^{1} \mathrm{M}$ Thelen*, 'M Garcia-Marquez, ' IJ Lehmann, 'D Keller, 'E Preugszat, ${ }^{2,3} \mathrm{M}$ von BergweltBaildon, 'HA Schlößer. 'Center for Molecular Medicine Cologne, Cologne, Germany; 2Department of Internal Medicine III, University Hospital, Ludwig Maximilians University, Munich, Germany; ${ }^{3}$ German Cancer Consortium (DKTK), Heidelberg, Germany

\subsection{6/jitc-2021-ITOC8.39}

Background Tumor-associated antigens (TAAs) and especially cancer testis antigens (CTAs) are classical tumor-specific targets for immunotherapies. As TAAs are shared between patients, strategies aiming to exploit these targets are scalable and potentially applicable across different types of cancer. Loss of target antigens and other mechanisms of immune escape have limited the success of CTA-directed immunotherapy. CAR T cells and other highly effective cellular therapies have renewed the interest in TAAs. Especially combined targeting of multiple antigens appears highly promising as recently shown in lymphoma. In our study, we aimed to characterize CTA-expression patterns and their impact on endogenous T-cell responses, T-cell abundance and antigen-presentation in esophago-gastric adenocarcinoma (EGA).

Materials and Methods 41 treatment-naïve EGA patients were included in our study. RNA of tumor and patient-matched healthy tissue was isolated and used for NanoString based RNA expression analysis of 26 CTAs and 25 genes associated with antigen-presentation. Based on CTA expression, 10 peptide pools were selected and co-cultured with peripheral blood mononuclear cells (PBMCs, $n=21$ ) to determine cellular anti-tumor immune responses in a FluoroSpot assay. Tcell abundance was assessed using immunohistochemistry (CD3, CD8) and digital image analyses of tumor area and invasive margin. Autologous CD40 activated B cells were used to expand antigen-specific T cells using peptide pools of CTAs.

Results NanoString analysis revealed pronounced differences regarding CTA expression, with CEP55 and MAGEA3/6 showing strong expression, while NY-ESO-1 or MAGEA1 were only weakly expressed. $68.3 \%$ (28/41) of the patients showed expression of $\geq 5 / 10$ analyzed TAAs simultaneously. In line with the frequent expression, $75.0 \%$ of the patients showed a cellular response against at least one of the TAAs. T-cell responses were most frequently detected to Survivin and NY-ESO-1 $(65.0 \%$ and $52.6 \%$ of patients, respectively), while only $20.0 \%$ responded to CEP55 or TTK peptide pools. Overall, 6/20 patients showed cellular responses against $\geq 5$ TAAs simultaneously. We found a strong correlation of T-cell abundance and antigen-presentation. In addition, patients with a high Immune-Score showed increased TAA expression. Finally, we demonstrate feasibility of TAAspecific T-cell expansion using CD40 activated B cells as potential strategy to induce or enhance TAA immune responses in EGA.

Conclusions Our study highlights the importance of TAAs in EGA. The identified antigens are highly relevant for immunomonitoring of clinical trials and as targets for immunotherapy. Personalized immunotherapeutic strategies targeting EGA-specific or even patient specific TAAs appear highly promising in this challenging disease.

Disclosure Information M. Thelen: None. M. Garcia-Marquez: None. J. Lehmann: None. D. Keller: None. E. Preugszat: None. M. von Bergwelt-Baildon: B. Research Grant (principal investigator, collaborator or consultant and pending grants as well as grants already received); Modest; Astellas, Roche, MSD. D. Speakers Bureau/Honoraria (speakers bureau, symposia, and expert witness); Modest; BMS. F. Consultant/Advisory Board; Modest; BMS. H.A. Schlößer: B. Research Grant (principal investigator, collaborator or consultant and pending grants as well as grants already received); Significant; Astra Zeneca.

\section{P06.06 ENHANCING TRAFFICKING AND RESISTANCE TO IMMUNOSUPPRESSION OF SYNTHETIC AGONISTIC RECEPTOR-TRANSDUCED T CELLS IN SOLID TUMOR MODELS}

${ }^{1,2} \mathrm{M}$ Schwerdtfeger*, ${ }^{1} \mathrm{M}$ Benmebarek, ${ }^{1} \mathrm{~F}$ Märkl, ${ }^{1} \mathrm{CH}$ Karches, ${ }^{1} \mathrm{~A}$ Öner, ${ }^{3} \mathrm{M}$ Geiger, ${ }^{1} \mathrm{~B}$ Cadilha, ${ }^{1} \mathrm{~S}$ Endres, ${ }^{2} \mathrm{~V}$ Desiderio, ${ }^{3} \mathrm{C}$ Klein, ${ }^{1,4,5} \mathrm{~S}$ Kobold. ${ }^{1}$ Center of Integrated Protein Science Munich (CIPS-M) and Division of Clinical Pharmacology, Department of Medicine IV, Klinikum der Universität München, LMU Munich, Member of the German Center for Lung Research (DZL), Munich, Germany; ${ }^{2}$ Department of Experimental Medicine, University of Campania 'Luigi Vanvitelli', Naples, Italy; ${ }^{3}$ Roche Innovation Center Zurich, Schlieren, Switzerland; ${ }^{4}$ German Center for Translational Cancer Research (DKTK), partner site Munich, Munich, Germany; ${ }^{5}$ Einheit für Klinische Pharmakologie (EKLiP), Helmholtz Zentrum München, German Research Center for Environmental Health (HMGU), Munich, Germany

\subsection{6/jitc-2021-ITOC8.40}

Background Chimeric antigen receptor therapy - although very efficacious in B cell malignancies - is facing many challenges which limit its success in solid tumors, e.g. on-target off-tumor toxicities, antigen heterogeneity, lack of $\mathrm{T}$ cell migration into tumors and an immunosuppressive tumor microenvironment. To better control on-target off-tumor effects and address antigen heterogeneity we developed a modular approach where we equipped $\mathrm{T}$ cells with a synthetic agonistic receptor (SAR). The SAR is only activated in the 